\title{
Personality Predictors of School Loneliness in Adolescent Students
}

\author{
Dorit Olenik-Shemesh \\ The Open University of Israel, Raanana, Israel
}

\author{
Moshe Zeidner \\ University of Haifa, Haifa, Israel
}

\begin{abstract}
This time-lagged study explores the role of the big-five factors of personality in predicting school loneliness in adolescent students. Personality data were gathered on 203 Israeli high-school students towards the beginning of the school year. Four months later, data were gathered on self-reported loneliness in school. Overall, these data support the conclusion that personality variables are meaningfully related to school-related loneliness in adolescents. Thus, consistent with our predictions, adolescents higher on extraversion and agreeableness were also lower on loneliness, whereas those higher on neuroticism were also higher on loneliness. A surprising positive relationship between openness and loneliness, when controlling for other personality factors, was found in the study, attributed to suppressor effects.
\end{abstract}

Keywords: personality, big-five factor, loneliness, adolescents

\section{Introduction}

Loneliness refers to a complex set of cognitions and affects reflecting the distressing and negative emotional experiences emanating from the individual's perceived deficiencies in intimate and social relationships (Ernst \& Cacioppo, 1999; Perlman, 1988; Weiss, 1973). Because people are social beings by nature, they require the support and friendship of others in their social environment throughout different stages of the life cycle — particularly during adolescence (Margalit \& Levin-Alyagon, 1994).

This study set out to explore the role of broad personality factors in predicting school loneliness in adolescent students. We begin by briefly discussing school loneliness among adolescent students. We then discuss a number of personality predictors of loneliness. Although we focus on school loneliness, we draw upon the broader literature on loneliness to gain a better understanding of how loneliness relates to broad personality variables and social variables in adolescent students.

\section{Adolescent Loneliness}

Adolescence has been characterized as one of the loneliest periods in one's life (Steinberg, Sullivan, \& Montoya, 1999), with feelings of loneliness particularly characteristic of adolescents and young adults (Getz, 2000; Goswick \& Jones, 1982; Margalit, 2010). Loneliness can be particularly difficult during adolescence, which is a time of individuation and of redefining one's own identity and social relationships. The pervasiveness of loneliness in adolescence is evident in its identification as a frequent complaint presented to adolescent hot-lines, health care centers and clinics, and youth counseling services (Rokach, Bauer, \& Orzeck,

Dorit Olenik-Shemesh, Ph.D., Department of Education and Psychology, The Open University of Israel. Moshe Zeidner, professor, Department of Counseling and Human Development, University of Haifa. 
2003).

The profound physical, social, and intellectual changes and transitions adolescents typically experience as part of their development trajectory may enhance their overall vulnerability and negatively impact upon their social self-image and feelings of loneliness (Coleman \& Hendry, 1990). Aside from these developmental transitions, striving for personal autonomy, the struggle for identity formation, individuation, and separation from parents, are among the factors which may contribute to adolescent loneliness (Brennan \& Alexandar, 1979; Coleman \& Hendry, 1990; Margalit, 2010). Whereas some studies have suggested that there are sex differences in loneliness (e.g., Weiss, 1973), these differences have been found to be negligible and have not been consistently replicated (e.g., Russell, Cutrona, McCrae, \& Gomez, 1980).

"School loneliness" refers to the perceived dissatisfaction with various aspects of social relationships in school settings (Krause-Parello, 2008; Margalit, 2010). Research on school-aged children and adolescents indicates that approximately $8 \%-12 \%$ experience feelings of loneliness in school (Asher \& Gazelle, 1999). In this paper, we conceptualize school loneliness as a subjective state of perceived social isolation in the school setting that accompanies the perception that one's social needs are not being met by the quality or quantity of one's intimate and social relationships in school (as cited in Cacioppo \& Patrick, 2008). More specifically, feelings of loneliness in the classroom may result from a perceived discrepancy between a student's desired level of intimacy and social relations at school and one's actual level of social relationship (Peplau \& Perlman, 1982). The greater the discrepancy between one's expectations and the perceived (or actual) states of one's social relations, the greater the feeling of loneliness and social dissatisfaction. Thus, some students may be alone at school but feel quite happy with that situation, while others may be involved in a large number of interpersonal relations yet are dissatisfied with important aspect of this relationship and experience loneliness (Russell, Cutrona, McCrae, \& Gomez, 2012).

There are a wide array of factors that may contribute to feelings of loneliness in school-aged adolescents. These include: limited social skills, lack of social support, poor communication skills, lacking qualities that determine classroom popularity (e.g., being successful, pretty or handsome, slim, athletic), social rejection and bullying by peers, changes in student's social environment (e.g., feeling isolated when making transition from one school to another), poor relations with parents, and disconnection to school personnel (see Krause-Parello, 2008, for a review).

In addition, developmental changes during adolescence mentioned above (e.g., need for autonomy, interdependence, relationships with same and opposite sex) can play a pivotal role in promoting loneliness. As young people enter their teenage years, peers become an increasingly important form of social support for adolescents. In fact, one's needs for intimacy, attachment, and acceptance are met more by peers than by the family. Indeed, adolescent students tend to be strongly peer-conscious and may be particularly sensitive to belonging to a school peer group during this period (Burgess, Ladd, Kochenderfer, Lambert, \& Birch, 1999; Goswick \& Jones, 1982). Therefore, persistent loneliness may be a particularly aversive experience for adolescents. A student lacking a close intimate friendship with peers at school may experience "emotional" loneliness, whereas a student lacking a valued peer group or social network to belong to may experience "social" loneliness (Weiss, 1973).

Since adolescents spend a good part of their daily life in the school setting, feeling lonely among one's classroom peers may pose a serious challenge to healthy social and emotional development for adolescents (Mahon, N. E. Yarcheski, A. Yarcheski, Cannnella, \& Banks, 2006). Thus, loneliness has been reported to be 
linked to a variety of different forms of individual and social pathology during adolescence and adulthood (e.g., delinquent behavior, alcoholism, suicide, depression, and somatic illness) as well as health-compromising behaviors (e.g., poor nutrition, unhealthy eating habits, unsafe sexual practices, lack of exercise; Mahon et al., 2006; McWhirter, 1990; Qualter, Brown, Munn, \& Rotenberg, 2010; Russell et al., 1980). Indeed, a body of developmental research suggests that lonely adolescents constitute "at risk" populations who may demonstrate social adjustment problems during adulthood (Asher, Hymel, \& Renshaw, 1984). In addition, an eight-year longitudinal study showed that childhood loneliness is a significant predictor of adolescent depression (Qualter, Brown, Munn, \& Rotenberg, 2010).

A meta-analytic study of personal correlates of loneliness during adolescence identified depression, shyness, and low self-esteem as strong correlates, and low social support and social anxiety as moderate correlates (Mahon et al., 2006). Also, sex differences appear to emerge by adolescence, with girls demonstrating lower loneliness than boys.

\section{Personality Factors in Loneliness}

Personality traits in adolescents might contribute to school loneliness in a number of different ways (Stokes, 1985). To begin with, certain personality traits (e.g., introversion, low agreeableness, hostility, and emotional reactivity) might make the adolescent student a less than desirable classmate, thus reducing available social relations as well as degrees of satisfaction with available peer relations at school. Second, personality variables may influence a student's perception and cognitive appraisals of and affective reactions to classroom situations, thus impacting the degree to which they feel supported, loved, and socially accepted at school. Third, certain broad personality characteristics might influence the way a student negotiates interpersonal interactions and conflicts in the classroom, thus making positive relationships difficult to maintain.

A number of broad personality factors have been found to be related to loneliness in emerging adult college students. Thus, research by Saklofske and Yackulic (1989) showed that extraversion (E), neuroticism (N), and psychoticism (P) of the Eysenck Personality Questionnaire (H. J. Eysenck \& S. B. G. Eysenck, 1975) contributed $45 \%$ and $37 \%$ of variance to loneliness scores of males and females respectively. Extraversion was inversely related to loneliness whereas neuroticism was significantly related to loneliness. Furthermore, earlier research by Saklofske, Yackulic, and Kelly (1986) showed extraversion was negatively correlated with social loneliness.

With respect to the FFM (five-factor model) of personality ("big-five"; Costa \& McCrae, 1980), neuroticism $(\mathrm{N})$, extraversion $(\mathrm{E})$, and $\mathrm{A}$ (agreeableness) are the factors that would be expected to be most robustly predictive of loneliness in general, and school loneliness in particular (Cheng \& Furnham, 2002; Saklofske \& Yackulic, 1989; Stokes, 1985). Thus, the various traits that comprise the neuroticism dimension (e.g., anxiety, sadness, hostility) are the very ones that may contribute to social isolation and loneliness (Costa $\&$ McCrae, 1980). The anxious and hostile characteristics of high neuroticism adolescents may make them unattractive as friends and they may reject the support of friends because they are especially fearful of being rejected by their classmates. Furthermore, the specific traits subsumed under extraversion (e.g., cheerful, energetic, optimistic, vivacious, and enthusiastic) and agreeableness (e.g., cordial, friendly, agreeable, peaceful, polite, kind, and tolerant) comprise the interpersonal sub-domain of personality assessment and would be expected to be positively related to richer social interactions and inversely related to adolescent loneliness. 
A meta-analytic study by Mahon et al. (2006) showed that loneliness is positively related to specific attributes of high neuroticism scores in adolescents (i.e., depression, anxiety, and low self-esteem). By contrast, extraversion was shown to be strongly negatively correlated with loneliness in adolescents. Regrettably, little is known about the predictive utility of other personality factors, such as openness, conscientiousness, and agreeableness, assessed in the currently prevalent FFM (McCrae \& John, 1992) of personality, with respect to loneliness in general, and school loneliness in particular. This study hopes to fill this needed gap.

With respect to gender differences on the big-five, a recent review by Marsh, Lüdtke, Muthén, Asparouhov, Morin, Trautwein, and Nagengast (2010) suggested that females tend to score higher than males on neuroticism and agreeableness, along with less consistent evidence that women score higher on extraversion (and conscientiousness); there is no clear support for evidence of gender differences in openness. A number of recent studies among adolescent populations, however, concur that females tend to score higher than males on all the big-five factors, particularly on neuroticism (Marsh et al., 2010; Donnellan \& Lucas, 2008; Schmitt, Realo, Voracek, \& Allik, 2008).

\section{Major Goals and Hypotheses}

As noted above, this study sets out to explore the role of broad personality in predicting adolescent school-based loneliness. This study hopes to improve upon a number of limitations of prior research. First, with respect to the breadth of coverage of broad personality factors, prior studies (Cheng \& Furnham, 2002; Saklofske \& Yackulic, 1989; Stokes, 1985) have relied largely on Eysenck's EPQ (Eysenck personality questionnaire), which taps only three broad personality factors, i.e., E, N, and P. As noted by Costa and McCrae (2011), these factors can hardly encompass the full range of individual differences variables. The currently prevalent FFM of personality is comprised of five factors: openness $(\mathrm{O})$, conscientiousness (C), extraversion $(E)$, agreeableness $(A)$, and neuroticism $(\mathrm{N})$. In the present study, we assessed the big-five factors as predictors of loneliness. In particular, we were interested in probing the role of agreeableness, which has not been tapped in previous studies, particularly in light of research suggesting that A (and E) are related to peer acceptance and positive interpersonal relations.

Second, with respect to participants, much of the available data concerns loneliness in emerging adult college students; more data are needed specifically regarding loneliness in adolescent students (Goswick \& Jones, 1982). Third, with respect to research design, prior research probing the relationship between broad personality predictors of loneliness (e.g., Cheng \& Furnham, 2002; Saklofske \& Yackulic, 1989; Stokes, 1985) has been largely based on cross-sectional research design. To correct this shortcoming, we examined personality factors several months prior to testing for loneliness. Fourth, few studies have included an external (objective) measure of loneliness in their study. This study includes a teacher report of loneliness of students in their classroom.

Based on theory and prior research, the following hypotheses were posited:

H1: The broad personality factors of extraversion and agreeableness will be negatively predictive of school loneliness in Israeli adolescent students;

$\mathrm{H} 2$ : The Neuroticism factor of the five factor model will be positively correlated with loneliness in Israeli adolescents;

H3: Adolescent girls in Israel will show higher mean levels of neuroticism, extraversion, and agreeableness, comparing to adolescent boys. 


\section{Method}

\section{Participants}

Data were collected on 203 (54\% female) high-school students enrolled in 14 high school classes in central Israel. Students ranged in age from 16 to 17 , with a mean age of $16.4(S D=0.51)$. The majority $(86 \%)$ of the students were native Israelis from middle class families. About three quarter of the students (76\%) came from intact homes, with the remainder brought up in single-parent families.

A multi-stage random cluster sampling procedure was employed to sample students for this study. During the first stage of sampling, four comprehensive high schools were randomly selected from among the pool of high schools located in the central Tel Aviv municipal district. During the second stage of sampling, fourteen 11th grade classrooms were randomly drawn from the four high schools sampled at stage 1 and students sampled from these classes.

In addition to students participants, homeroom teachers responsible for each of the abovementioned 14 classrooms sampled for this study, were asked to evaluate each of the students in their respective homeroom with respect to his or her social functioning in the classroom along a 5-point Likert scale $(1=$ "Very poor", $5=$ "Very good").

\section{Measures}

Personality traits. Personality traits, modeled after the FFM, were assessed via a Hebrew adaptation (Zeidner, 2002) of the 60-item OCEANIC (openness/conscientiousness/extraversion/agreeableness/ neuroticism/index condensed scale). The OCEANIC (Roberts, 2001) is a short version of the NEO-PI-R (NEO personality inventory_revised) questionnaire and was developed to operationalize the "big-five" personality factors, a thoroughly researched and long-standing robust model of personality (McCrae \& John, 1992). Participants rate their agreement with 60 statements on a five-point scale. The scale provides no facet scores, rather it is purely a measure of the following five factors of the "big-five": openness ( e.g., "I have thought about the origins of the universe", alpha $=0.83$ ); conscientiousness (e.g., "I would describe myself as efficient", alpha $=0.84$ ); extraversion (e.g., "I like to be where there is lots of action", alpha $=0.80$ ); agreeableness ("I like to be kind to everyone I know", alpha =0.81); neuroticism ("I get upset easily", alpha $=0.84$ ). The scale reliabilities observed in the present study were comparable to those reported by Roberts (2001), which ranged between 0.80 and 0.90 . Although initially designed for college students and adults, the OCEANIC has been successfully employed with adolescents in the Israeli context in prior studies (e.g., Zeidner \& Shani-Zinovich, 2011). Further evidence supporting the scale's validity may be found in Roberts (2001).

Loneliness. Loneliness was operationalized by the Hebrew adaptation of the Illinois loneliness and social dissatisfaction scale (Asher, Hymel, \& Renshaw, 1984; Williams \& Asher, 1992). This 24-item questionnaire was developed to assess children's feelings of loneliness and social dissatisfaction with respect to the school context. The 16 primary items focused on children's feelings of loneliness (e.g., "I'm lonely"), feelings of social adequacy versus inadequacy (e.g., "I'm good at working with other children"), or subjective estimations of peer status (e.g., "I have lots of friends"). The other eight filler items focused on children's hobbies or preferred activities (e.g., "like to paint and draw"; "I watch TV a lot"). Children responded to each of the 24 items by indicating on a five-point scale how much each statement was a true description of themselves (i.e., always true, true most of the time, true sometimes, and hardly ever true). Prior research showed that items loaded on one single Loneliness factor, and had good internal consistency reliability (Asher et al., 1984). Thus, only one total 
sum score was employed in this study, with higher scores indicating higher levels of loneliness. The alpha reliability coefficient for this sample (alpha $=0.92)$ compared favorably to that reported in the literature.

Teacher-based adjustment inventory. In order to obtain independent assessments of students' social integration in the classroom teachers the 14 homeroom teachers rated each of the participants in their respective classes with respect to their level of social integration in the classroom ( $1=$ "Very poor", $5=$ "Very good").

Demographic data. Demographic data were gathered by asking students to complete an inventory that included questions about their gender, age, and parents' marital and educational and economic status.

\section{Procedure}

Students who submitted parental consent forms completed the OCEANIC, a measure of the five-factor model of personality, towards the beginning of the school year. About four months later, students completed measures of loneliness and teachers rated students in their classroom with respect to social functioning.

\section{Results}

\section{Preliminary Analyses}

Preliminary analysis, using SPSS (Statistical Package for Social Science) Linear Mixed Models procedure, indicated that the between-classroom variance accounted for $5 \%$ of the total variance in loneliness. When considering the borderline ICC (intra-class correlation coefficient) (ICC $=0.05$ ), coupled with the relatively small number of level two factors (14 classroom), we decided to use ordinary least square regression analysis in this study rather than random coefficient modeling.

Bivariate correlations. Correlations for all variables and descriptive statistics (Means, $S D$ ) are shown in Table 1. As predicted, both extraversion $(r=-0.46)$ and agreeableness $(r=-0.38)$ were negatively and moderately correlated with school-based loneliness, whereas neuroticism $(r=0.20)$ was positively, but modestly, correlated with loneliness. A comparison of dependent correlations indicated that both extraversion ( $t$ $=-7.09, p<0.001)$ and agreeableness $(t=-6.07, p<0.001)$ are more strongly correlated with loneliness than neuroticism.

As shown in Table 1, teacher-rated social classroom integration was significantly correlated with school-based loneliness, $r_{(200)}=-0.39, p<0.001$. Thus, students higher on loneliness were also rated by their teacher as being less integrated into the classroom than their less lonely counterparts. This lends convergent validity to the student self-report measure of school loneliness we employed in this study.

Table 1

Intercorrelation Matrix for Key Measures

\begin{tabular}{|c|c|c|c|c|c|c|c|}
\hline Measure & 1 & 2 & 3 & 4 & 5 & 6 & 7 \\
\hline 1. Openness & 1.00 & $0.19^{*}$ & $0.16^{*}$ & 0.13 & $0.17^{*}$ & 0.06 & 0.02 \\
\hline 2. Conscientiousness & & 1.00 & 0.07 & $0.28^{*}$ & 0.14 & -0.14 & 0.10 \\
\hline 3. Extraversion & & & 1.00 & $0.45^{*}$ & -0.10 & $-0.46^{*}$ & $0.17^{*}$ \\
\hline 4. Agreeableness & & & & 1.00 & -0.08 & $-0.38^{*}$ & $0.15^{*}$ \\
\hline 5. Neuroticism & & & & & 1.00 & $0.20^{*}$ & -0.09 \\
\hline 6. Loneliness & & & & & & 1.00 & $-0.37^{*}$ \\
\hline 7. Teacher assessment & & & & & & 1.00 & 1.00 \\
\hline Means & 3.48 & 3.99 & 3.96 & 4.59 & 3.08 & 24.10 & 3.72 \\
\hline$S D s$ & 0.86 & 0.78 & 0.75 & 0.64 & 0.87 & 4.09 & 1.12 \\
\hline
\end{tabular}

Note. ${ }^{*} p<0.05$ and beyond. 


\section{Gender Differences}

Table 2 presents descriptive statistics, by gender, on the key variables. A MANOVA for the effects of gender on all the five-factor scales of the FFM showed a significant effect, $F_{(5,191)}=3.87, p<0.002$, with gender accounting for about $9 \%$ of the vector variable variance. Consistent with our hypotheses and past research, adolescent girls scored higher than boys on neuroticism, $3.24(0.84)>2.86(0.87), t_{(195)}=-3.05, p<$ $0.003, d=-0.45$, by the order of almost half a standard deviation. Furthermore, girls scored significantly higher than boys, on average, on agreeableness, $4.68(0.65)>4.47(0.65), t_{(195)}=-2.33, p<0.02, d=-0.35$ and extraversion, $4.05(0.74)>3.83(0.76), t_{(195)}=-1.99, p<0.05, d=-0.28$.

Table 2

Descriptive Statistics for Key Variables in the Study, by Gender

\begin{tabular}{|c|c|c|c|c|c|c|}
\hline \multirow[t]{2}{*}{ Key variables } & \multicolumn{3}{|c|}{ Adolescent boys } & \multicolumn{3}{|c|}{ Adolescent girls } \\
\hline & $M$ & $S D$ & $M$ & $S D$ & $t$ & $d$ \\
\hline \multicolumn{7}{|l|}{ 1.Personality } \\
\hline Openness & 3.47 & 0.85 & 3.49 & 0.87 & -0.09 & -0.02 \\
\hline Conscientiousness & 3.93 & 0.77 & 4.04 & 0.79 & -1.02 & -0.15 \\
\hline Extraversion & 3.83 & 0.76 & 4.05 & 0.74 & $-1.99^{*}$ & -0.28 \\
\hline Agreeableness & 4.47 & 0.65 & 4.68 & 0.62 & $-2.33^{*}$ & -0.35 \\
\hline Neuroticism & 2.86 & 0.87 & 3.24 & 0.84 & $-3.05^{*}$ & -0.45 \\
\hline 2. Classroom loneliness & 0.95 & 0.65 & 1.81 & 0.62 & 1.18 & 0.16 \\
\hline 3. Teacher's assessment ${ }^{a}$ & 3.74 & 1.12 & 3.70 & 1.19 & 0.81 & 0.04 \\
\hline
\end{tabular}

Notes. Male $n$ 's varied from 82 to 85 ; female $n$ 's varied from 112 to 115 . Mean comparisons are based on independent $t$-tests. $D$-scores based on Hedge's unbiased sigma scores. Homeroom teacher's assessment of student social is functioning in the classroom.

\section{Regression Analysis}

We employed regression analysis to determine the utility of personality in predicting loneliness, statistically controlling for the effects of gender. With the big-five personality variables entering into the equation, and gender serving as covariate, the significant model, $F_{(6,189)}=13.05, p<0.001$, accounted for $29 \%$ of the loneliness variance, $R S Q=0.293, S E=0.54, \triangle R S Q=0.29$. Four of the five personality variables made a significant contribution to the model: extraversion, $B=-0.37, t=-5.40, p<0.001$; agreeableness, $B=-0.20, t=$ 2.72, $p<0.007$; neuroticism, $B=0.13, t=2.02, p<0.04$; and openness, $B=0.14, t=2.20, p<0.03$. Thus, consistent with our predictions, adolescents higher on extraversion and agreeableness were also lower on loneliness, whereas those higher on neuroticism were also higher on loneliness. The positive relationship between openness and loneliness, when controlling for other personality factors, was surprising.

\section{Discussion}

Overall, these data support the conclusion that personality variables are meaningfully related to school-related loneliness in adolescents. Whether considered separately or in combination with other big-five factors, extraversion and agreeableness were inversely related to loneliness. These data support De Raad's (2011) observation that the facets and specific traits that comprise extraversion (e.g., cheerful, energetic, optimistic, vivacious, and enthusiastic) and agreeableness (e.g., cordial, friendly, agreeable, peaceful, polite, kind, and tolerant) comprise the interpersonal sub-domain of personality assessment, and by implication, are thus inversely related to loneliness. 
These data are highly consistent with recent meta-analytic research showing that extraversion is strongly negatively correlated with loneliness in adolescents (Mahon et al., 2006). Our data are also consistent with those reported by Jensen-Campbell (2002) showing that both agreeableness and extraversion were positively associated with peer acceptance and friendship in middle school children. Furthermore, given that low agreeableness has been identified as a primary factor of the psychoticism scale of the Eysenck personality questionnaire (Eysenck, 1991), our data concur with earlier studies showing that psychoticism is positively correlated with loneliness. It stands to reason that adolescents who are more extraverted are characterized as people-oriented, sociable, and easy-going, with a substantial need for stimulation (Cheng \& Furnham, 2002). Thus, they manifest the very behaviors that increase richer social and interpersonal contacts, which, in turn, reduce the likelihood of experiencing loneliness. By contrast, the more withdrawn, shy, reserved, bookish, behaviors of introverts may limit their opportunities to form intimate relationships with others, thereby leading to fewer and less satisfying relationships in their social networks.

As predicted, neuroticism was positively, though modestly, related to loneliness (Cheng \& Furnham, 2002; Saklofske \& Yackulic, 1989; Stokes, 1985). These results are consistent with prior meta-analytic research by Mahon et al. (2006) showing that loneliness is positively related to specific characteristics of high neuroticism scores, such as depression, anxiety, and low self-esteem. Overall, these data suggest that adolescents who are predisposed to worry and to focus on what can go wrong may also be predisposed to see themselves as alone, isolated, and lonely.

Following Costa and McRae (1980), the various traits that comprise the neuroticism dimension are the very ones that contribute to human discontent and social isolation and loneliness. Specifically, their heightened attention to and biased cognitive processing of self-related information could lead to an evaluation of being a failure in interpersonal situations, further exacerbating the perceived loneliness and accompanying anxiety. In addition, the overly emotional, moody, anxious, and worrying adolescent may be more susceptible and sensitive to relational deficits or may have a limited capacity to enjoy satisfying relations (Saklofske \& Yackulic, 1989). These lonely emotionally unstable adolescents may have a subjective theory of the world that says that people cannot be trusted. Because other people are frequently perceived as threatening, this makes social interactions more stressful. Furthermore, high neuroticism scoring adolescent scores may protect themselves from the anxiety of interpersonal relations by withdrawing from their peers and being convinced that they are not lovable and fearful of rejection, reject potential friends and intimates in order to protect themselves from rejection (Stokes, 1985).

Attachment theory may shed some additional light on mechanisms through which neuroticism may impact on loneliness in adolescents. Thus, a massive literature (Mikulincer \& Shaver, 2007) demonstrates that anxious attachment styles are associated with how people negotiate social relationships and regulate emotions during times of stress. In addition, prior research (Noftle \& Shaver, 2006) has suggested that the broad trait of neuroticism is consistently correlated with anxious attachment. It follows, that adolescents who are high on neuroticism may also be characterized by fear of rejection and abandonment, and by doubts about one's desirability as a relationship partner. This, in turn, complicates the formation and maintenance of social relationships, increasing the chances of social isolation and loneliness. $\mathrm{N}$ may be a particularly relevant factor for romantic relationships that are first established during the adolescent years.

There is solid evidence from longitudinal studies that neuroticism predicts vulnerability to future stress outcomes (Matthews, Deary, \& Whiteman, 2003). Intriguingly, there are at least two paths to vulnerability 
revealed by longitudinal studies of emotional responses to life events (e.g., Ormel \& Wohlfarth, 1991). More neurotic individuals react to events with elevated, possibly excessive negative effect. In addition, neuroticism predicts future adverse events; it seems that behaviors typical of the trait such as interpersonal arguments increase the likelihood of high neuroticism scorers experiencing life events. To some degree, these individuals bring stress on themselves. More fine-grained investigations demonstrate several mediating processes that contribute to stress vulnerability (Zeidner \& Matthews, 2011).

Given the negligible correlation between openness and loneliness, how can we account for the significant positive effect of openness in contributing to loneliness variance in the sequential regression model? It is plausible that a suppression effect may be at play here, with the variables in the predictor stock suppressing some of the irrelevant variance in openness. Thus, high openness adolescents, who may be especially creative, independent in their thinking, and highly competent, may find it more difficult to fit in with their conventional peer group and therefore experience greater loneliness. Additional research is needed to test this speculative account.

\section{Gender Differences}

Adolescent boys and girls were not found to be reliably differentiated on mean loneliness scores. These findings are consistent with a body of literature suggesting that gender differences tend to be negligible and have not been consistently replicated (e.g., Russell et al., 1980).

Furthermore, our data suggest that adolescent girls, on average, tend to be higher on agreeableness, extraversion, and neuroticism, on average, when compared to adolescent boys. These data concur with our hypothesis and a recent review by Marsh et al. (2010) showing that females tend to score higher than males in relation to neuroticism and agreeableness, along with less consistent evidence that women score higher on extraversion (and conscientiousness); there is no clear support for evidence of gender differences in openness. A number of recent studies among adolescent populations, however, concur that women tend to score higher than men on all the big-five factors, particularly on neuroticism (Marsh et al., 2010; Donnellan \& Lucas, 2008; Schmitt et al., 2008).

The reader should keep in mind the following limitations of the present study. First, the personality and loneliness measures used were self-report; some of the observed relationships may be accounted for by common method variance. Second, we employed a unidimensional scale of loneliness that did not allow us to differentiate among various facets (cognitive vs. emotional), forms (romantic, familial, and friendships community), durations (episodic, situational, and chronic), displays (aggressive vs. passive), and etiologies (social neglect, and social rejection) of adolescent loneliness. Future research might benefit by doing so. Third, the time interval in our prospective design between data collection on personality factors and loneliness was restricted; future research should examine longer intervals.

\section{Conclusions}

Overall, the data suggest that key personality factors are predictive of classroom loneliness in adolescent students. Although we cannot infer causality with these correlational data, these results could be informative when conceptualizing intervention or prevention program in schools to foster healthy social relations and reduce loneliness among adolescent students. Students high on extraversion and agreeableness and high on Neuroticism might be at risk for experiencing loneliness and should be monitored and considered for primary 
prevention psychological programs.

Furthermore, in light of prior research showing significant patterns of relationships between loneliness and both social skills and social support (Cheng \& Furnham, 2002), future intervention could help students by improving their social skills and by building supportive and satisfying relationships with peers in the classroom. The school environment can also be structured in ways that promote acceptance and friendship and satisfaction with support provided. Adolescent can be taught critical social interaction skills and how to foster friendships. Because boys seem to report poorer social skills than girls and also be less satisfied with support received from classmates, specific interventions targeted to meet the needs of adolescent boys might be especially helpful to their well being.

\section{References}

Asher, S. R., \& Gazelle, H. (1999). Loneliness, peer relations, and language disorder in childhood. Topics in Language Disorders, 19, 16-33.

Asher, S. R., Hymel, S., \& Renshaw, P. D. (1984). Loneliness in children. Child Development, 55, 1457-1464.

Brennan, T., \& Alexandar N. (1979). Adolsecent loneliness. Bolder, Colorado: Behavioral Research Institute.

Burgess, K. B., Ladd, G. W., Kochenderfer, B. J., Lambert, S. F., \& Birch, S. H. (1999). Loneliness during early childhood: The role of interpersonal behaviors and relationships. In K. J. Rotenberg, \& S. Hymel (Eds.), Loneliness in childhood and adolescence (pp. 109-134). New York: Cambridge University Press.

Cacioppo, J. T., \& Patrick, B. (2008). Loneliness: Human nature and the need for social connection. New York: W. W. Norton \& Co..

Cheng, H., \& Furnham, A. (2002). Personality, peer relations, and self-confidence as predictors of happiness and loneliness. Journal of Adolescence, 25, 327-339.

Coleman, J. C., \& Hendry, L. (1990). Adolescence and society: The nature of adolescence (2nd ed.). Florence, K.Y.: Taylor \& Frances/Routledge.

Costa, P. T., \& McCrae, R. R. (1980). Influence of extraversion and neuroticism on subjective well-being: Happy and unhappy people. Journal of Personality and Social Psychology, 38, 668-678.

Costa, Jr., P. T., \& McCrae, R. R. (2011). The NEO personality inventory NEO-PI-R. In G. Boyle, D. Saklofske, \& G. Matthews (Eds.), Handbook of personality assessment (Vol. II, pp. 423-446). Beverly Hills, C.A.: Sage.

De Raad, B. (2011). Structural models of personality. In P. J. Corr, \& G. Matthews (Eds.), Cambridge handbook of personality psychology (pp. 127-147). Cambridge: Cambridge University Press.

Donnellan, M. B., \& Lucas, R. E. (2008). Age differences in the big five across the life span: Evidence from two national samples. Psychology and Aging, 23, 558-566.

Ernst, J. M., \& Cacioppo, J. T. (1999). Lonely hearts: Psychological perspectives on loneliness. Applied \& Preventive Psychology, $8,1-22$.

Eysenck, H. J. (1991). Dimensions of personality: 16, 5 or 3? Criteria for a taxonomic paradigm. Personality and Individual Differences, 12, 773-790.

Eysenck, H. J., \& Eysenck, S. B. G. (1975). Manual of the Eysenck personality questionnaire. London: Hodder \& Stoughton.

Getz, G. E. (2000). A novel social situation and loneliness. Psychological Reports, 86, 947-950.

Goldstein, A. P., Sprakfin, R. P., Gershae, N. J., \& Klein, P. (1980). Skill streaming the adolescents. Champaign, I.L.: Research Press.

Goswick, R. A., \& Jones, W. H. (1982). Components of loneliness during adolescence. Journal of Youth and Adolescence, 1, 373-383.

Jensen-Campbell, L. (2002). Agreeableness, extraversion, and peer relations in early adolescence: Winning friends and deflecting aggression. Journal of Research in Personality, 36, 224-251.

Krause-Parello, C. A. (2008). Loneliness in the school setting. The Journal of School Nursing, 24, 66-70.

Mahon, N. E., Yarcheski, A., Yarcheski, Th. J., Cannella, B. L., \& Banks, M. M. (2006). A meta-analytic study of predictor for loneliness during adolescence. Nursing Research, 55, 308-315. 
Margalit, M. (2010). Lonely children and adolescents: Self-perceptions, social exclusion and hope. New York: Springer.

Margalit, M., \& Levin-Alyagon, M. (1994). Learning disability subtyping, loneliness, and classroom adjustment. Learning Disability Quarterly, 17, 297-310.

Marsh, H. W., Lüdtke, O., Muthén, B., Asparouhov, T., Morin, A. J. S., Trautwein, U., \& Nagengast, B. (2010). A new look at the big five factor structure through exploratory structural equation modeling. Psychological Assessment, 22, 471-491.

Matthews, G., Deary, I. J., \& Whiteman, M. C. (2003). Personality traits (2nd. ed.). New York: Cambridge University Press.

McCrae, R. R., \& John, O. P. (1992). An introduction to the five-factor model and its applications. Journal of Personality, 60, $175-215$

McWhirter, B. T. (1990). Loneliness: A review of the literature with implications for counseling and research. Journal of Counseling and Development, 68, 417-422.

Mikulincer, M., \& Shaver, P. R. (2007). Attachment in adulthood: Structure, dynamics, and change. New York: Guilford Press.

Noftle, E. E., \& Shaver, P. R. (2006). Attachment dimensions and the big five personality traits: Associations and comparative ability to predict relationship quality. Journal of Research in Personality, 40, 179-208.

Ormel, J., \& Wohlfarth, T. (1991). How neuroticism, long-term difficulties, and life situation change influence psychological distress: A longitudinal model. Journal of Personality and Social Psychology, 60, 744-755.

Peplau, L. A. \& Perlman, D. (1982). Perspectives on loneliness. In L. A. Peplau, \& D. Perlman (Eds.), Loneliness: A sourcebook of current theory, research, and therapy (pp. 1-18). New York: Wiley.

Perlman, D. (1988). Loneliness: A life-span, family perspective. In R. M. Milardo (Ed.), New perspectives on family: Families and social networks (pp. 190-220). Thousand Oaks, C.A.: Sage Publications.

Qualter, P., Brown, S. L., Munn, P., \& Rotenberg, K. J. (2010). Childhood loneliness as a predictor of adolescent depressive symptoms: An 8-year longitudinal study. European Child \& Adolescent Psychiatry, 19, 493-501.

Roberts, R. (2001). Openness, conscientiousness, extraversion, agreeableness, neuroticism index condensed (OCEANIC): Preliminary technical reports draft 2. Sydney: University of Sydney Press.

Rokach, A., Bauer, N., \& Orzeck, T. (2003). The experience of loneliness of Canadian and Czech youth. Journal of Adolescence, 26, 267-282.

Russell, D., Peplau, L. A., \& Cutrona, C. E. (1980). The revised UCLA loneliness scale: Concurrent and discriminant validity evidence. Journal of Personality and Social Psychology, 39, 472-480.

Russell, D. W., Cutrona, C. E., McCrae, C., \& Gomez, M. (2012). Is loneliness the same as being alone? Journal of Psychology, 146, 7-22.

Saklofske, D. H., Yackulic, R. A., \& Kelly, I. W. (1986). Personality and loneliness. Personality and Individual Differences, 7 , 899-901.

Saklofske, D. H., \& Yackulic, R. A. (1989). Personality predictors of loneliness. Personality and Individual Differences, 10, 467-472.

Schmitt, D. P., Realo, A., Voracek, M., \& Allik, J. (2008). Why can't a man be more like a woman? Sex differences in big five personality traits across 55 cultures. Journal of Personality and Social Psychology, 94, 168-182.

Steinberg, A. G., Sullivan, V. J., \& Montoya, L. A. (1999). Loneliness and social isolation in the workplace for deaf individuals during the transition years: A preliminary investigation. Journal of Applied Rehabilitation Counseling, 30, 22-30.

Stokes, J. P. (1985). The relation of social network and individual difference variables to loneliness. Journal of Personality and Social Psychology, 48, 981-990.

Weiss, R. S. (1973). Loneliness: The experience of emotional and social isolation. Cambridge, M.A.: MIT Press.

Williams, G. A., \& Asher, S. R. (1992). Assessment of loneliness at school among children with mild mental retardation. American Journal on Mental Retardation, 96, 373-385.

Zeidner, M. (2002). Hebrew adaptation of the OCEANIC: Laboratory for research on personality and individual differences. Haifa: University of Haifa.

Zeidner, M., \& Gerald, M. (2011). Anxiety 101. New York: Springer.

Zeidner, M., \& Shani-Zinovich, I. (2011). Do academically gifted and non-gifted students differ on the big-five and on adaptive status? Some recent data and conclusions. Personality and Individual Differences, 50, 641-645. 